\begin{tabular}{|l|l|l|}
\hline$j+r .5$ & Pago iof \\
DEC 061992 & 1. EDT 623543 \\
\hline
\end{tabular}

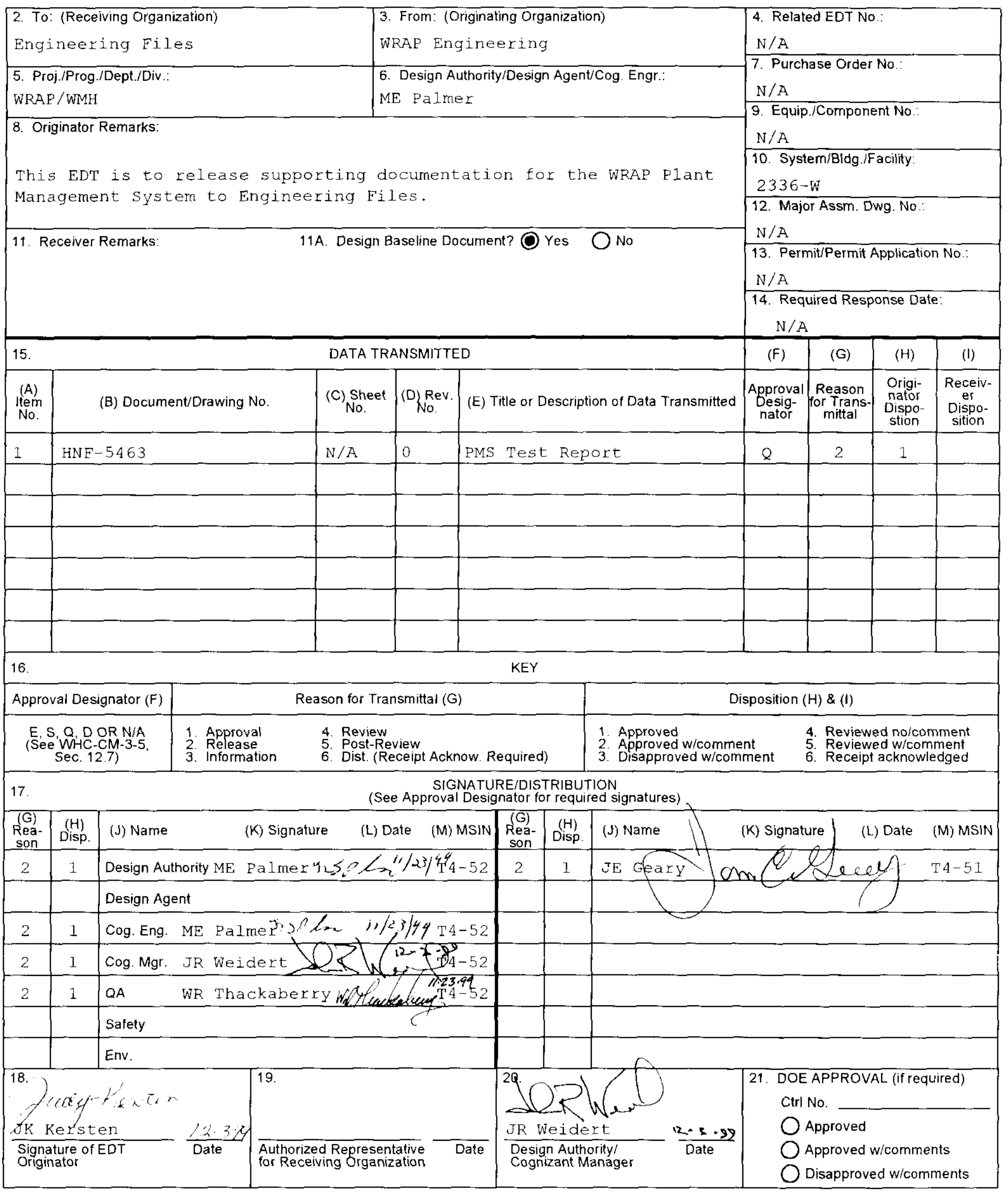




\section{WASTE RECEIVING AND PROCESSING FACILITY PLANT MANAGEMENT SYSTEM TEST REPORT}

\section{ME Palmer}

Waste Management Federal Services of Hanford, Inc., Richland, WA 99352 U.S. Department of Energy Contract DE-AC06-96RL13200

EDT/ECN: $\quad$ EDT-623543 UC: 506

Org Code: $32600 \quad$ Charge Code: AJ60

B\&R Code: EW02J16 Total Pages: 23

Key Words: WRAP, PMS, PMS-01

Abstract: This document contains the testing results for installation of a new server in the WRAP facility.

** PLC is a registered trademark of Rockwell International Corporation, Pittsburgh, PA.

** Oracle is a registered trademark of Oracle Corporation, World Headquarter, Redwood Shores, CA.

TRADEMARK DISCLAIMER. Reference herein to any specific comercial product, process, or service by trade name, trademark, manufacturer, or otherwise, does not necessarily constitute or imply its endorsement, reconmendation, or favoring by the United States Government or any agency thereof or its contractors or subcontractors.

Printed in the United States of America. To obtain copies of this document, contact: Document Control services, P.0. BOX 950, Mailstop H6-08, Richland WA 99352, Phone (509) 372-2420; fax (509) 376-4989.
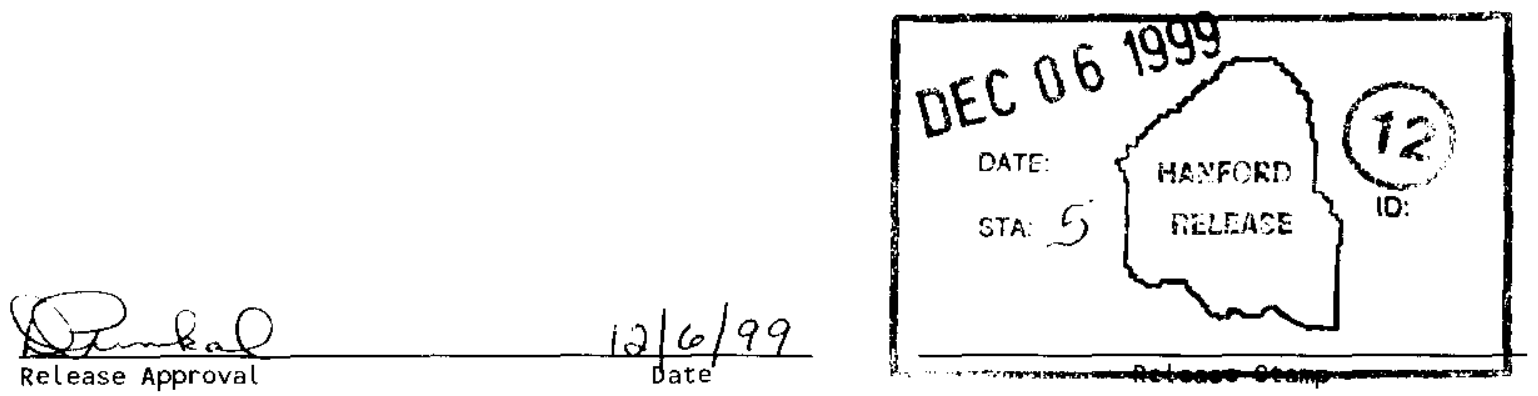

\section{Approved for Public Release}


HNF-5463, Rev. 0

\section{PLANT MANAGEMENT SYSTEM TEST REPORT}

\section{November, 1999}

Test plan HNF-4351 defines testing required for installation of a new server in the WRAP facility. Test Procedure PMS-01 was issued as HNF-4622, Rev. 0 based on this test plan. The Test Procedure was later revised and issued as Rev. 1. This document is the completed test report for Test Procedure PMS-01. 
HNF-5463, Rev. 0

This Page Intentionally left Blank 

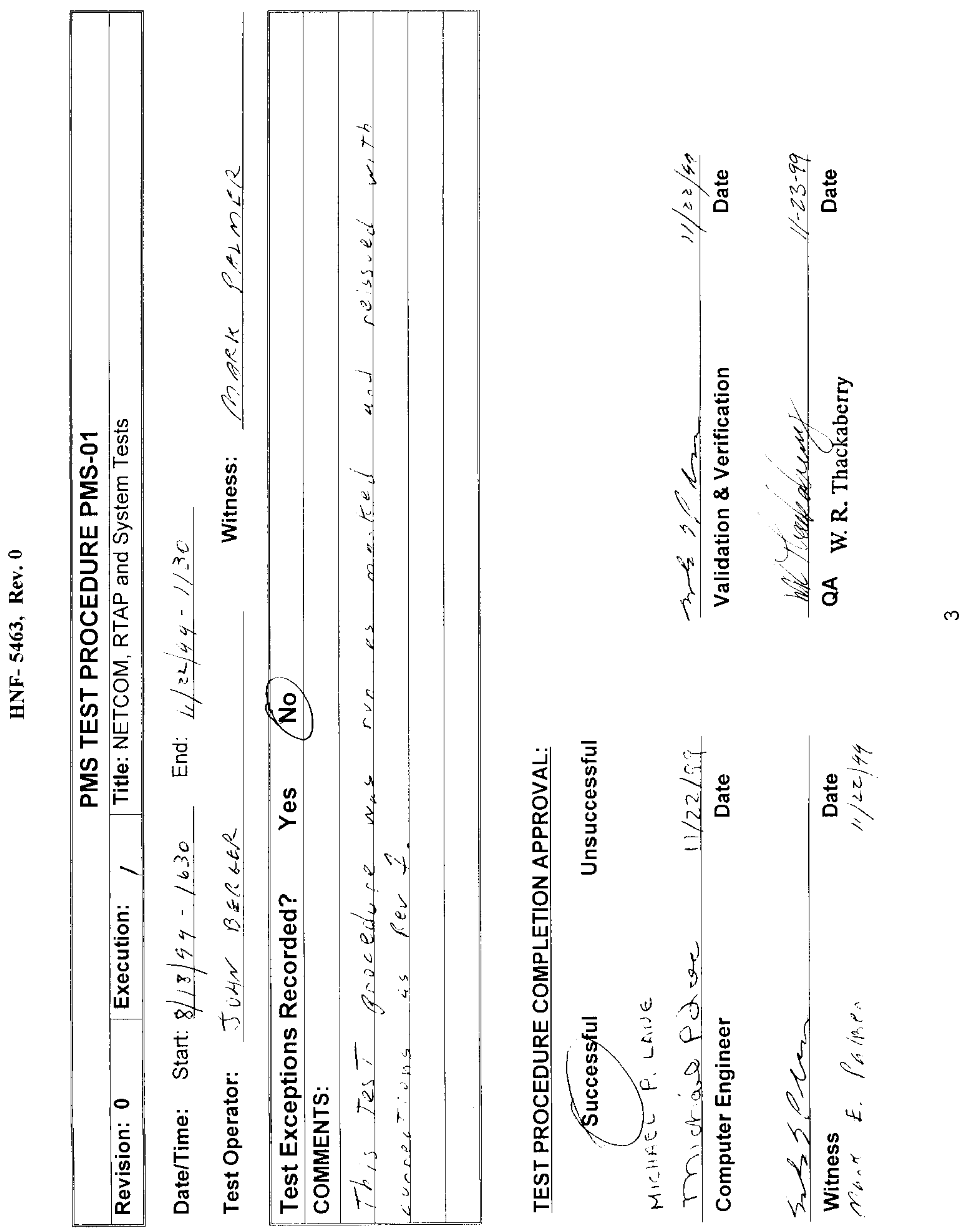


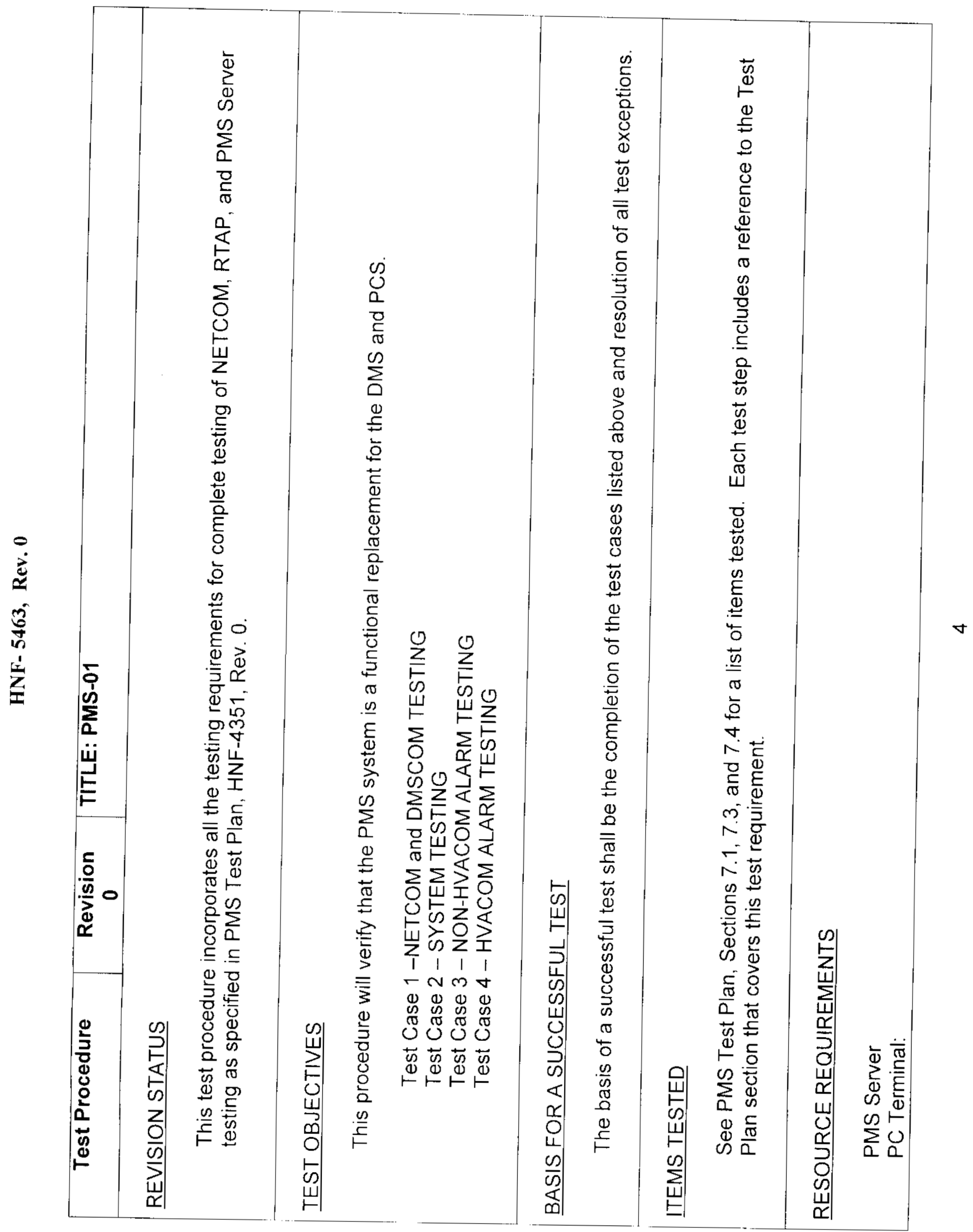




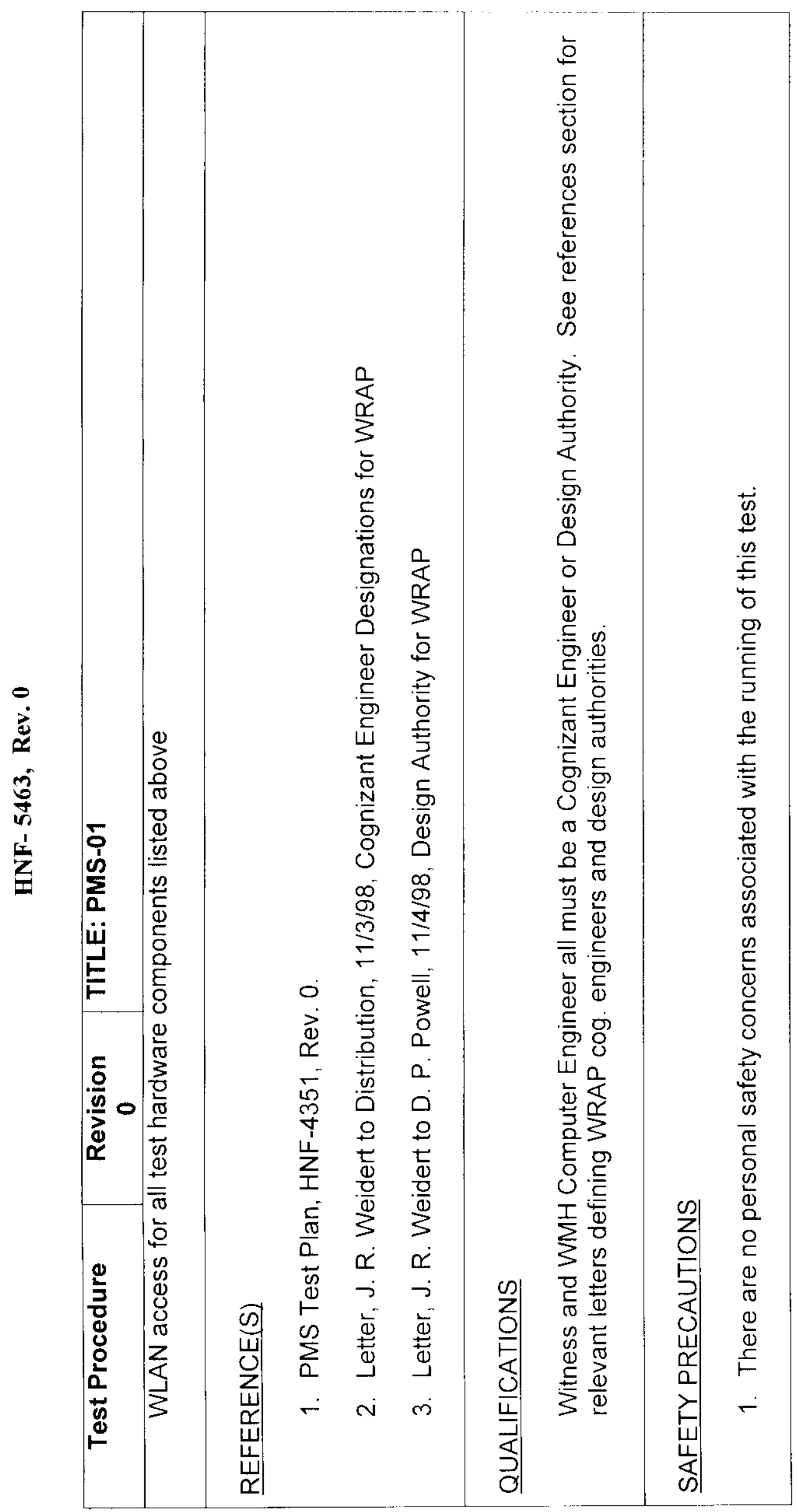




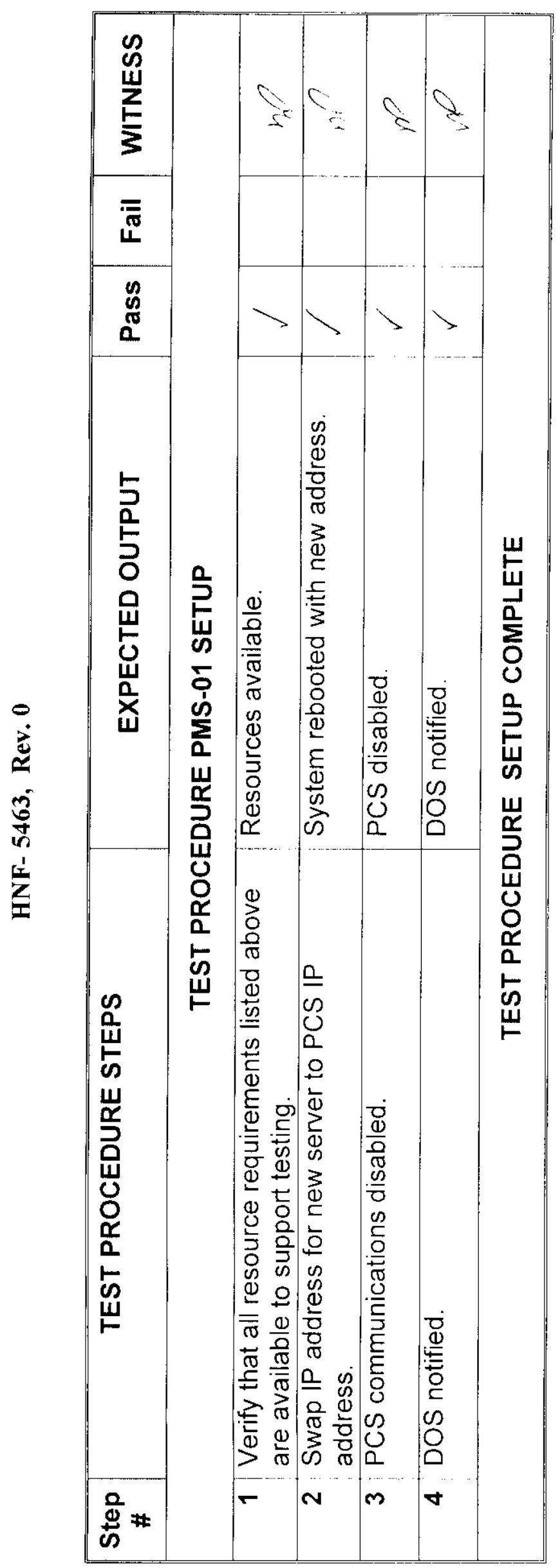




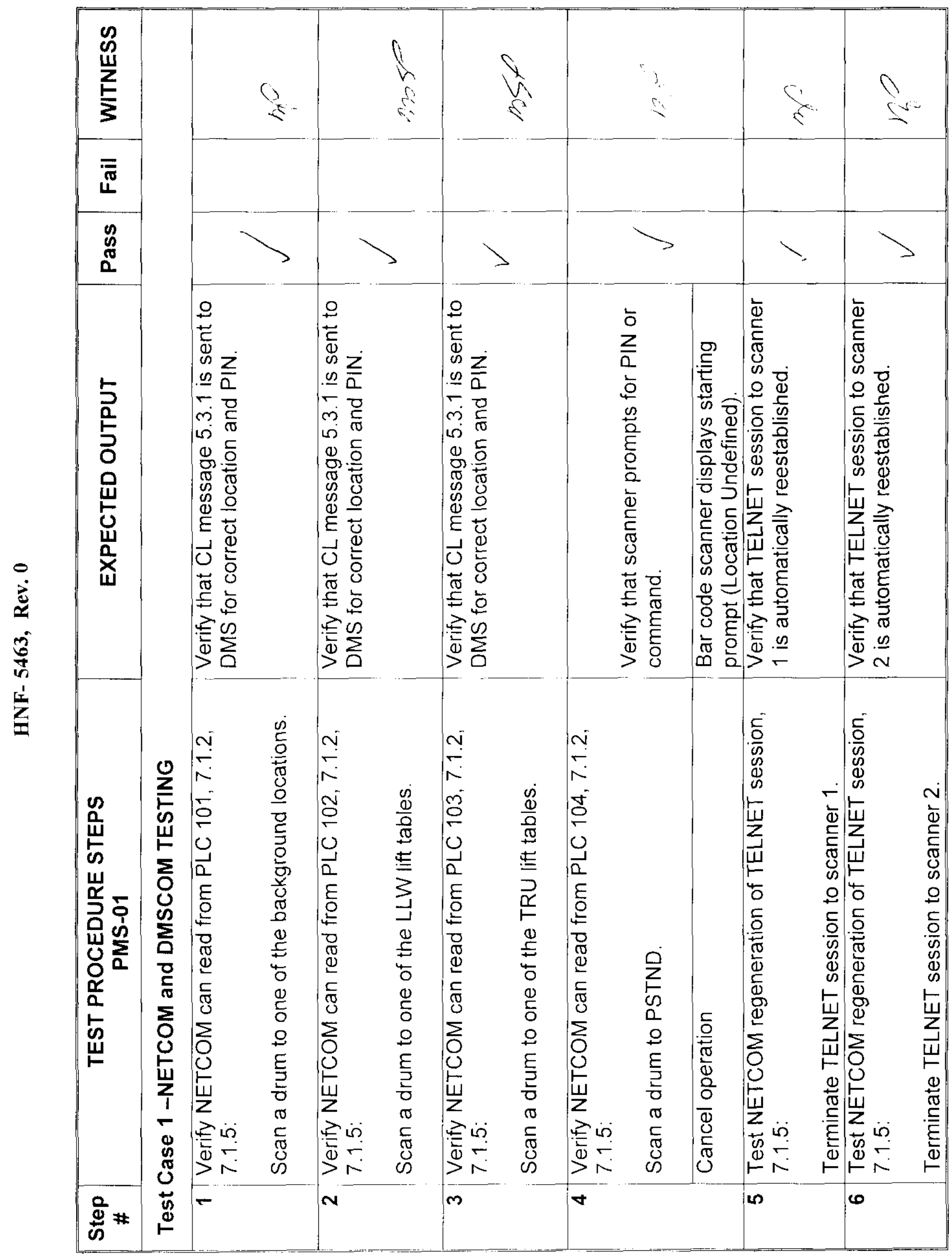




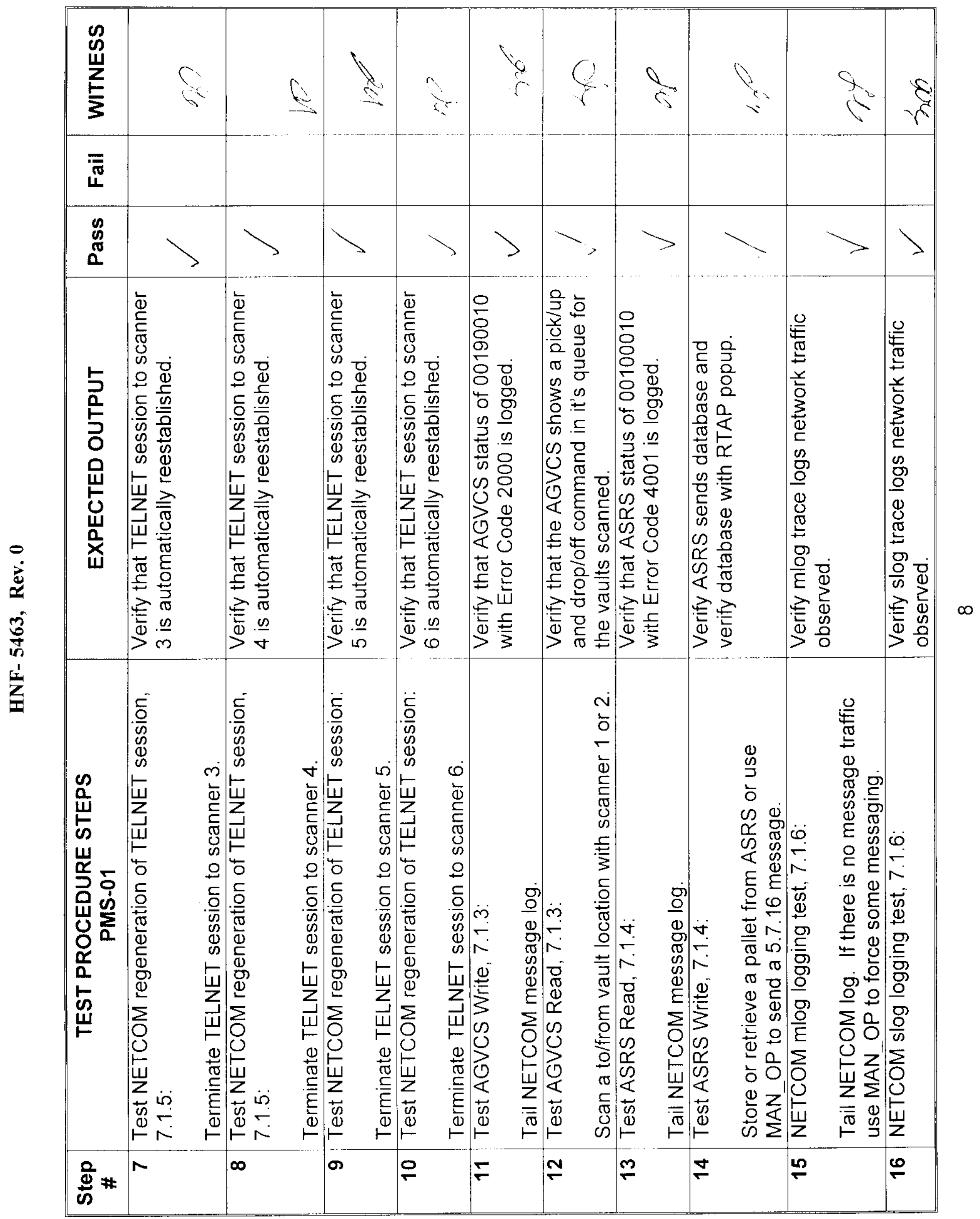




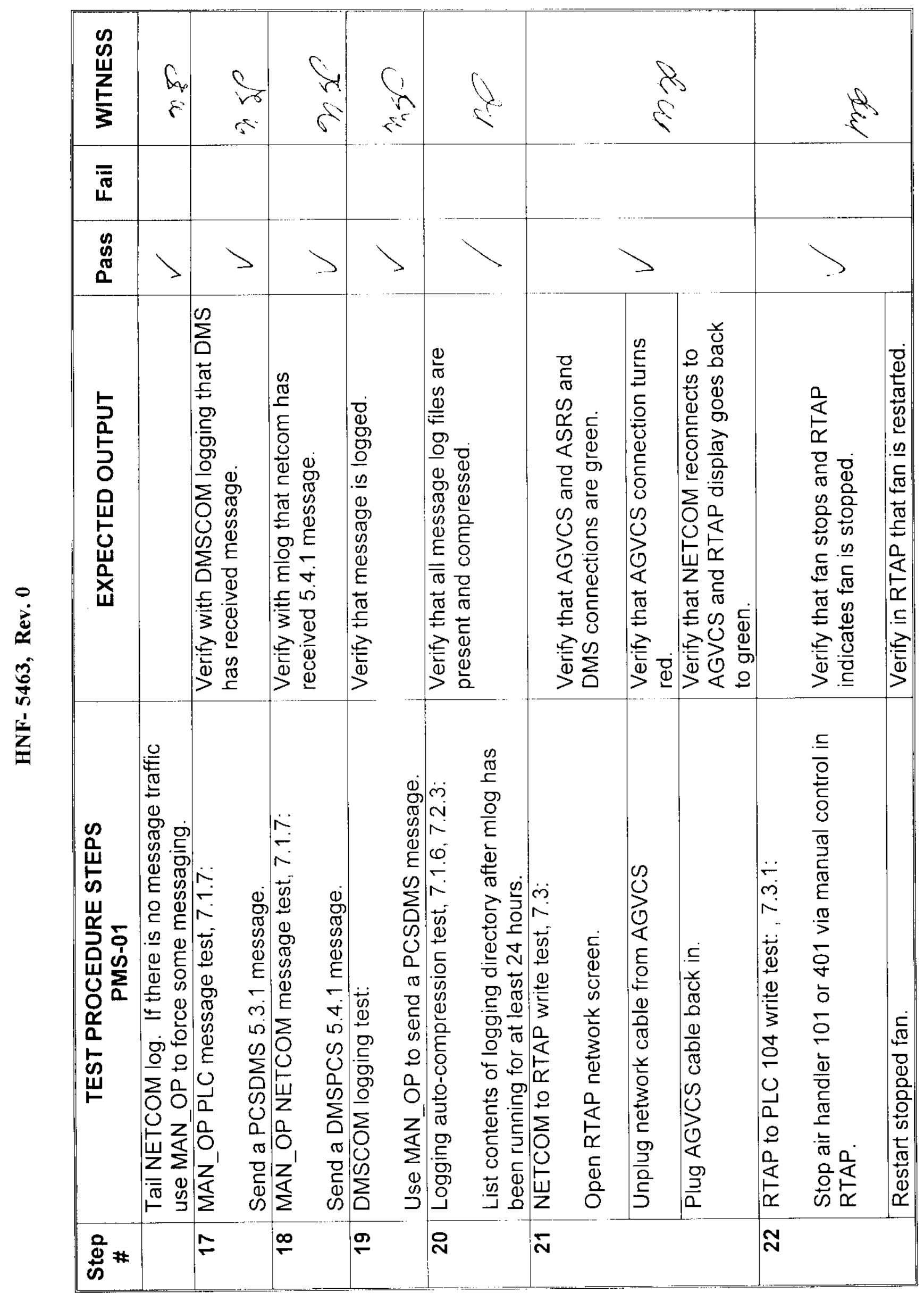




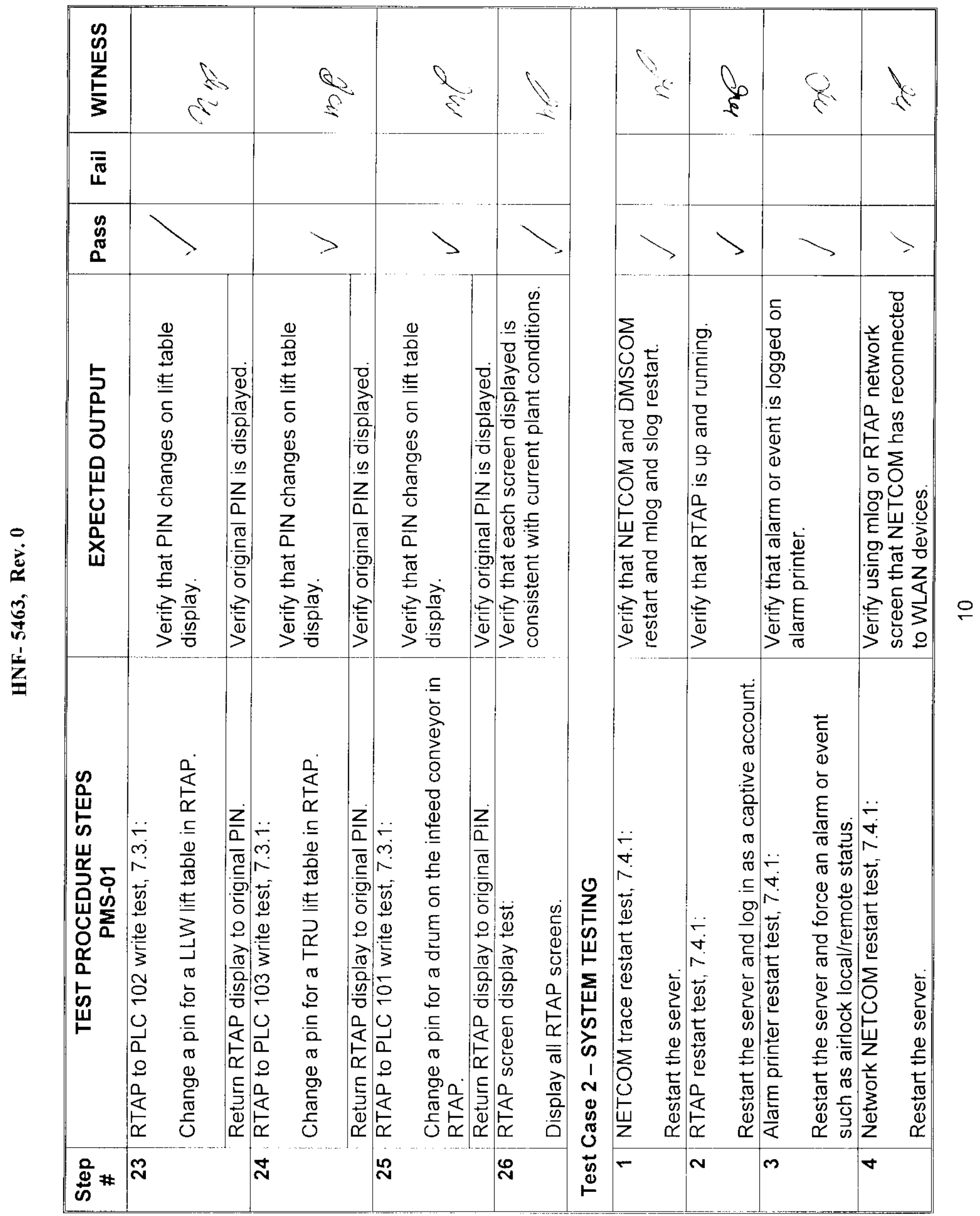




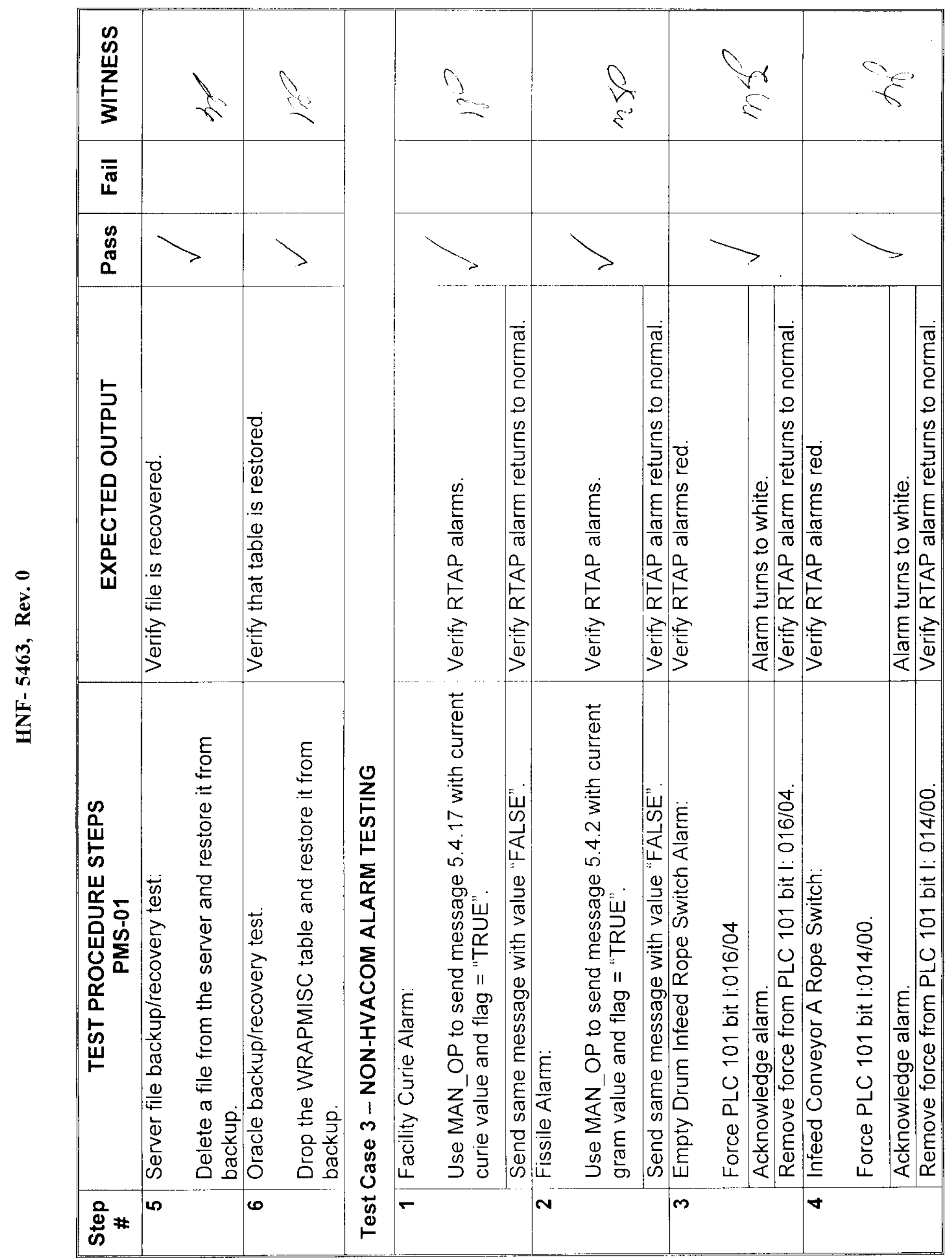




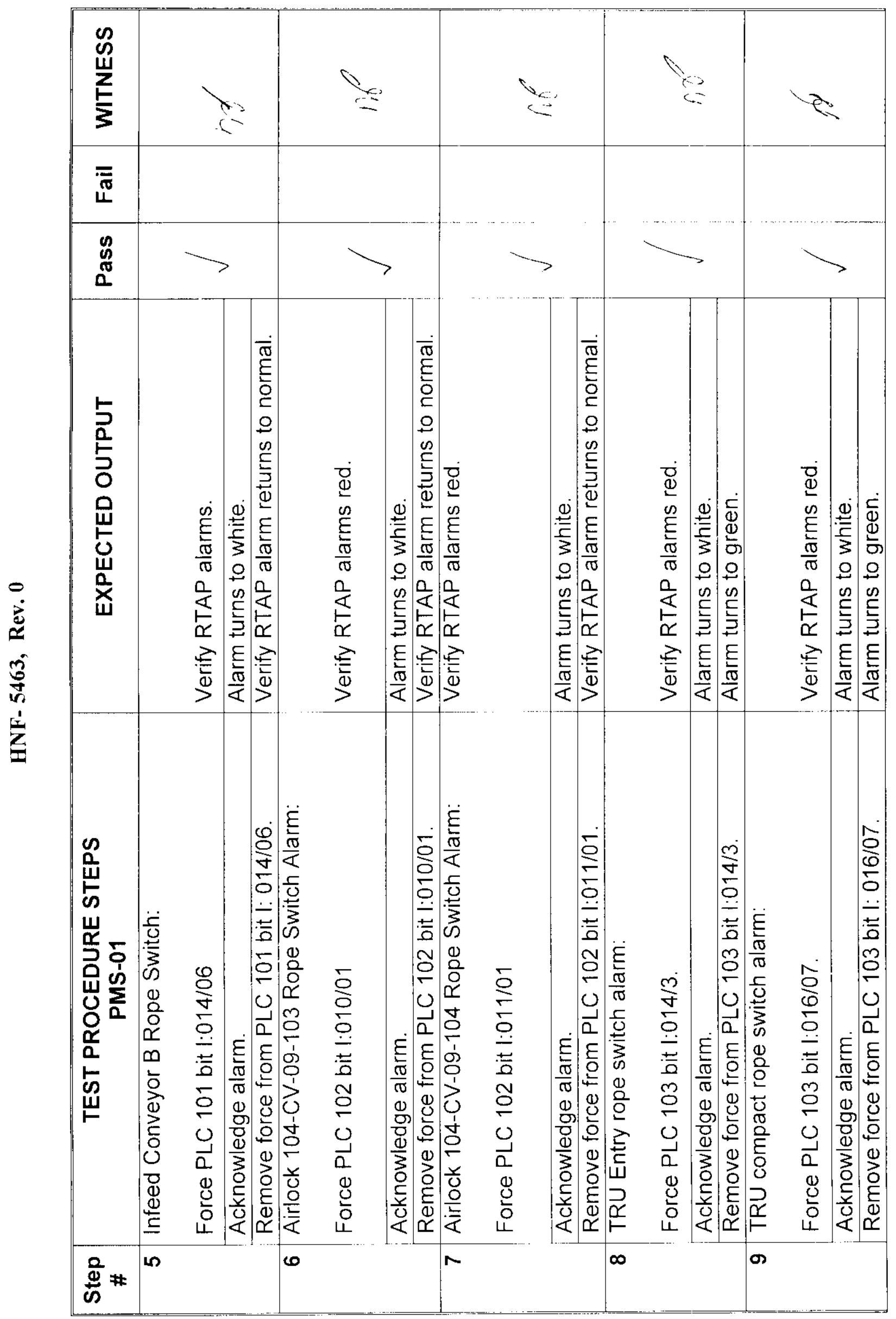




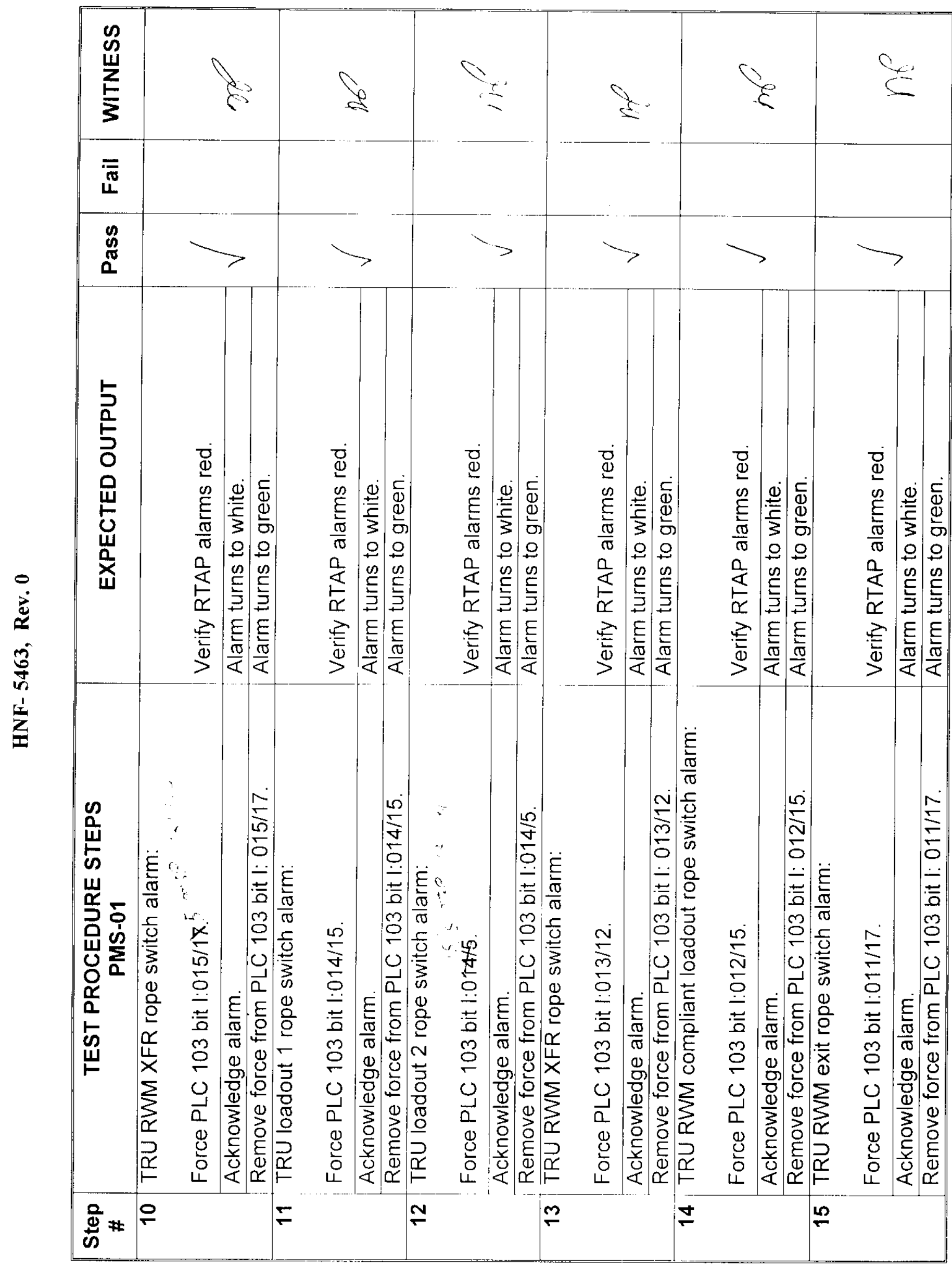




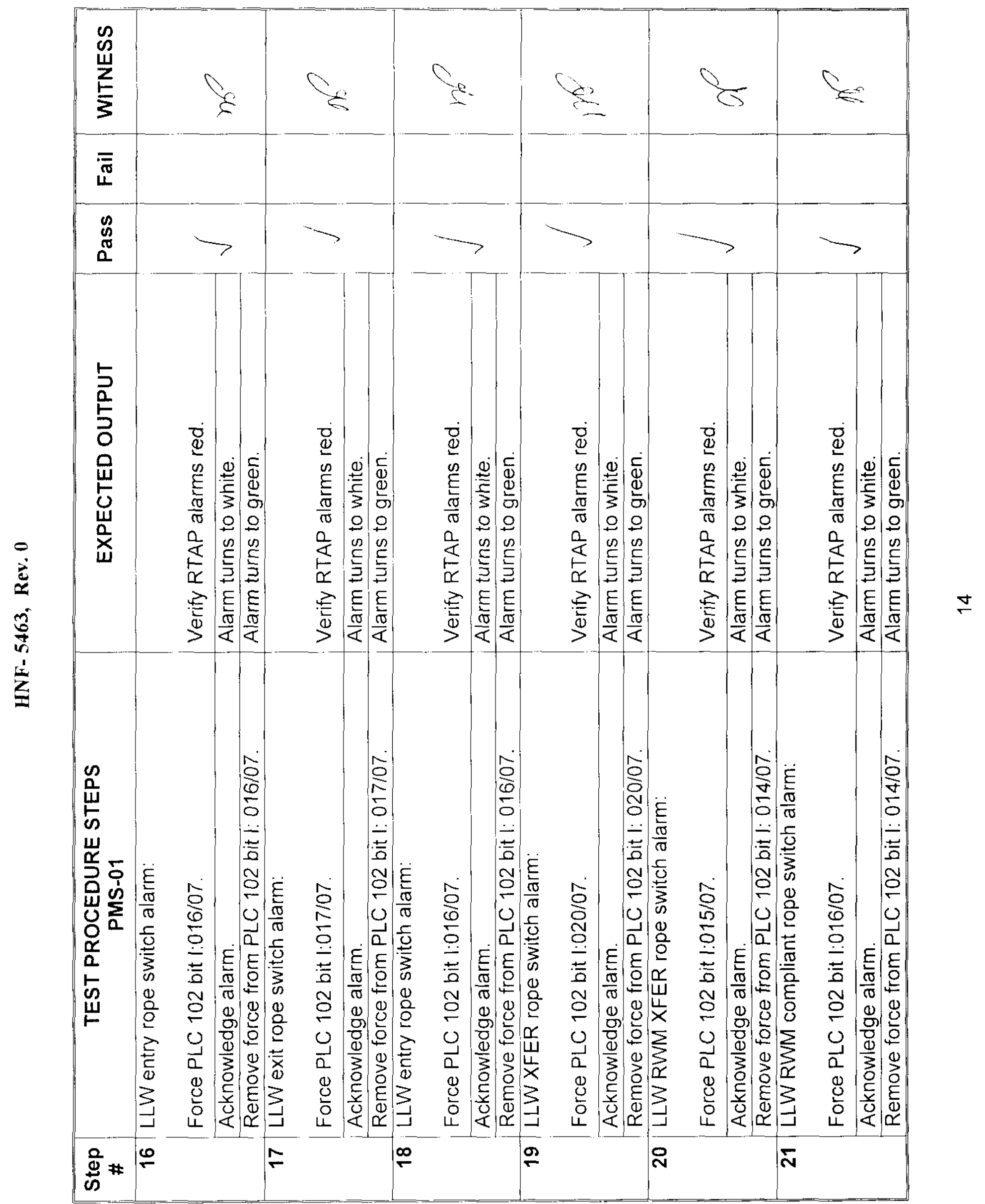




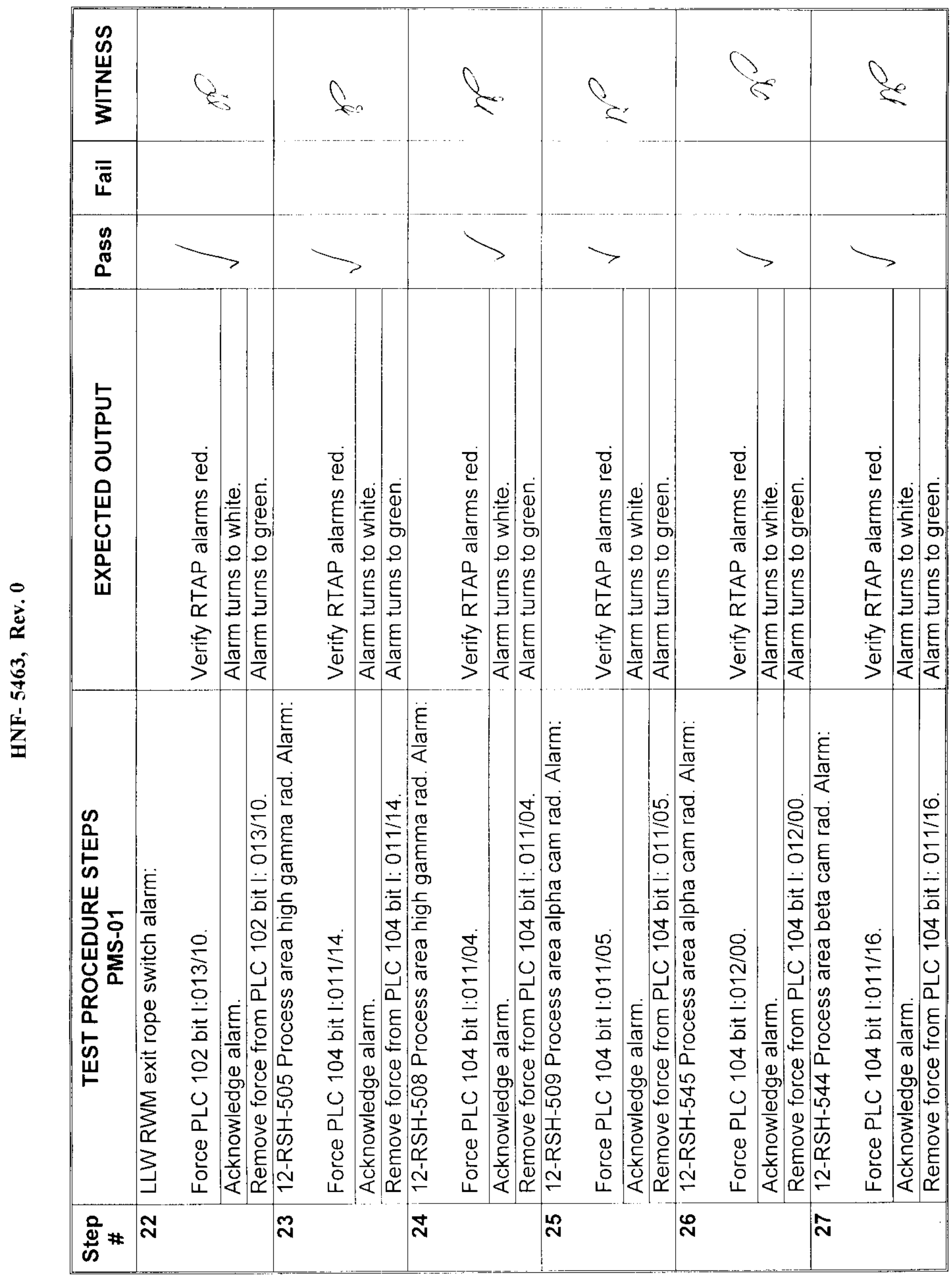




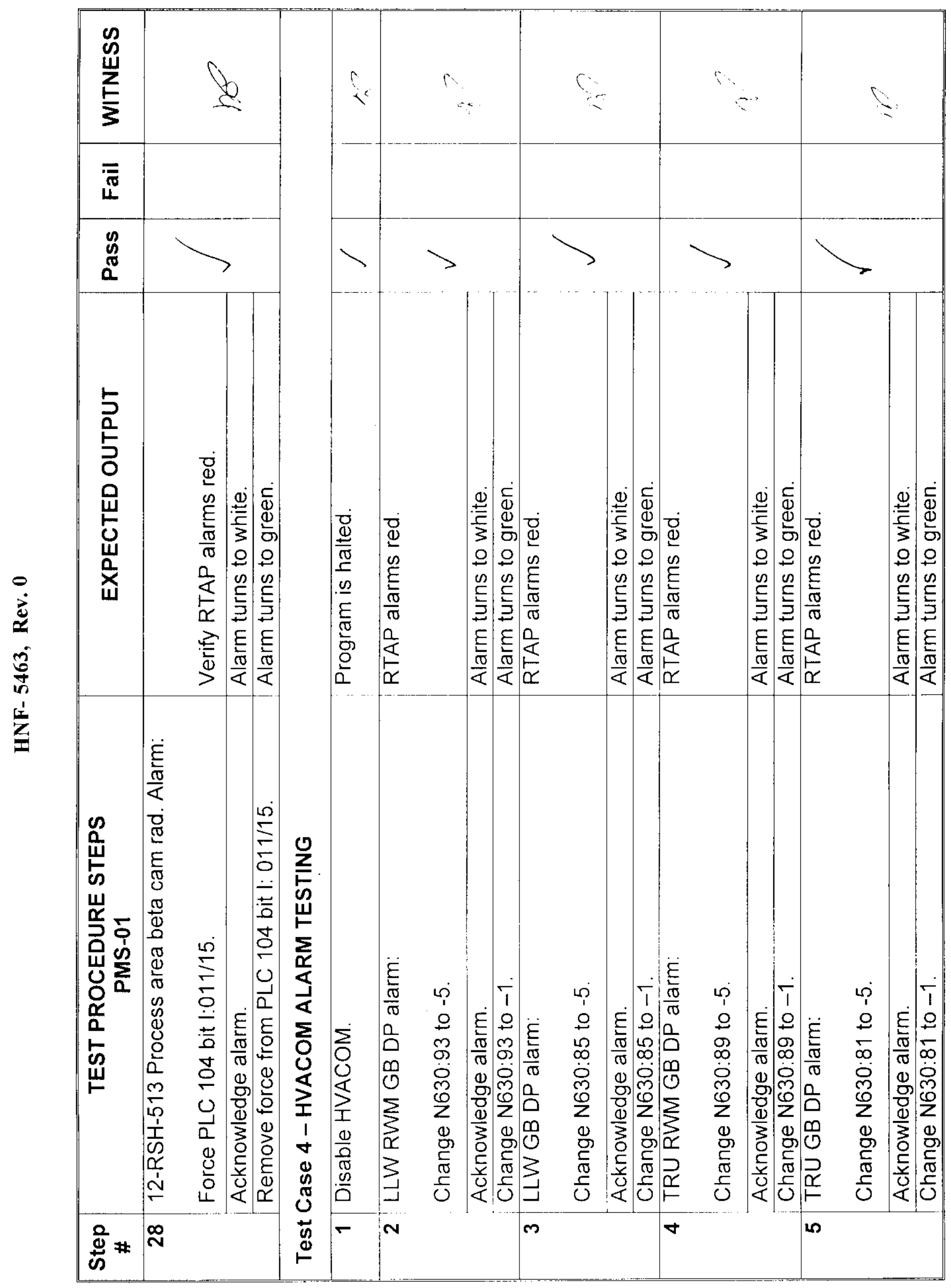




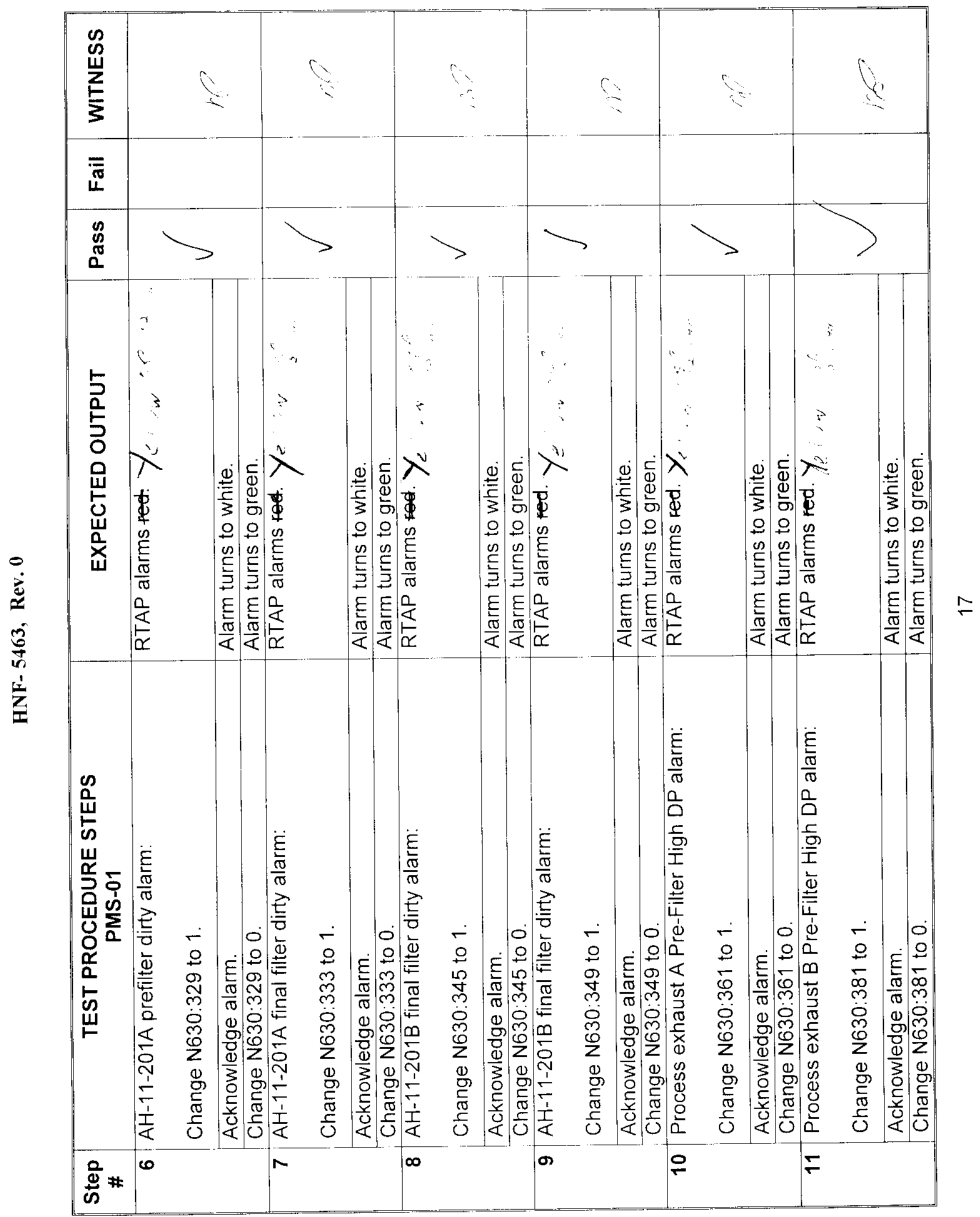




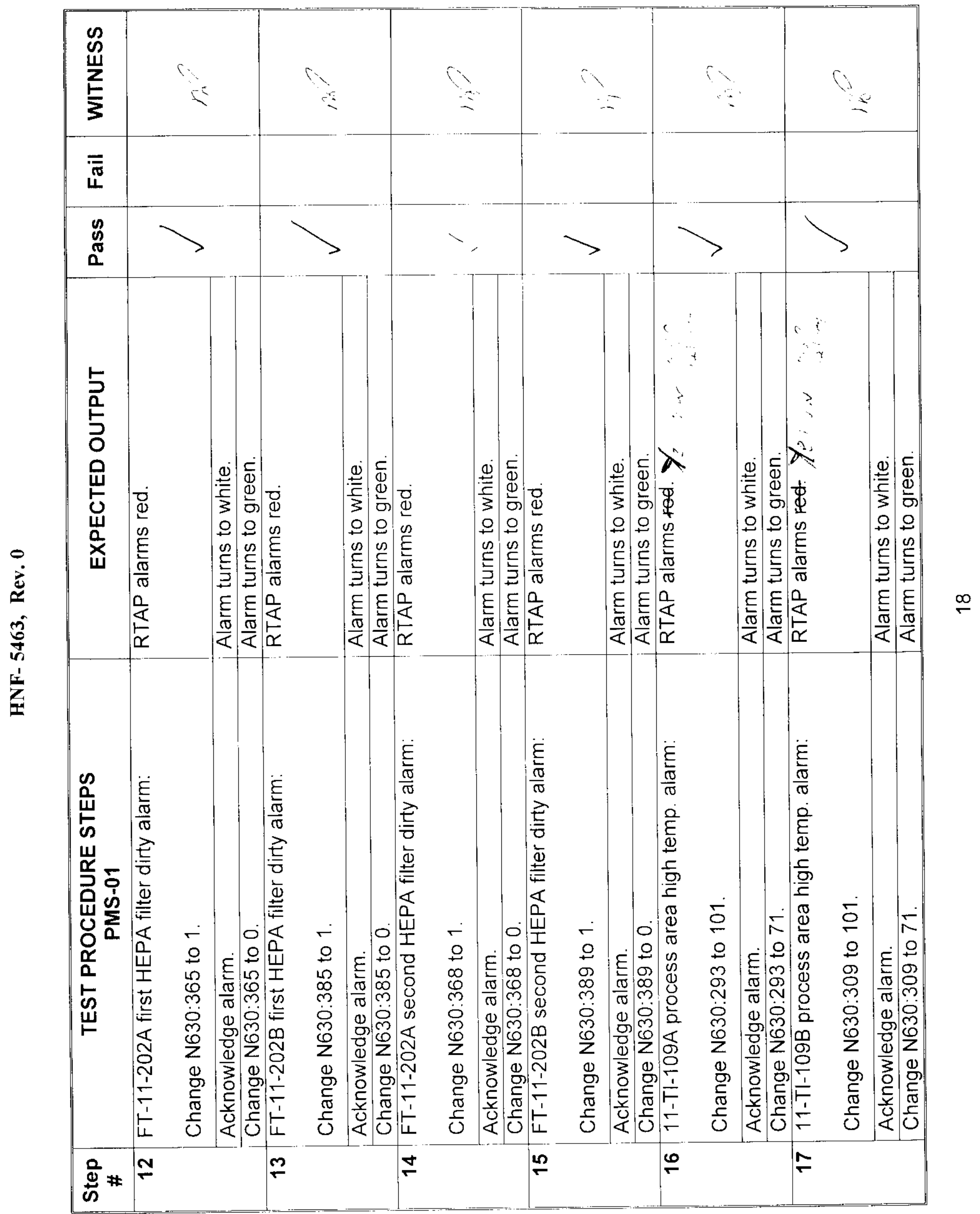




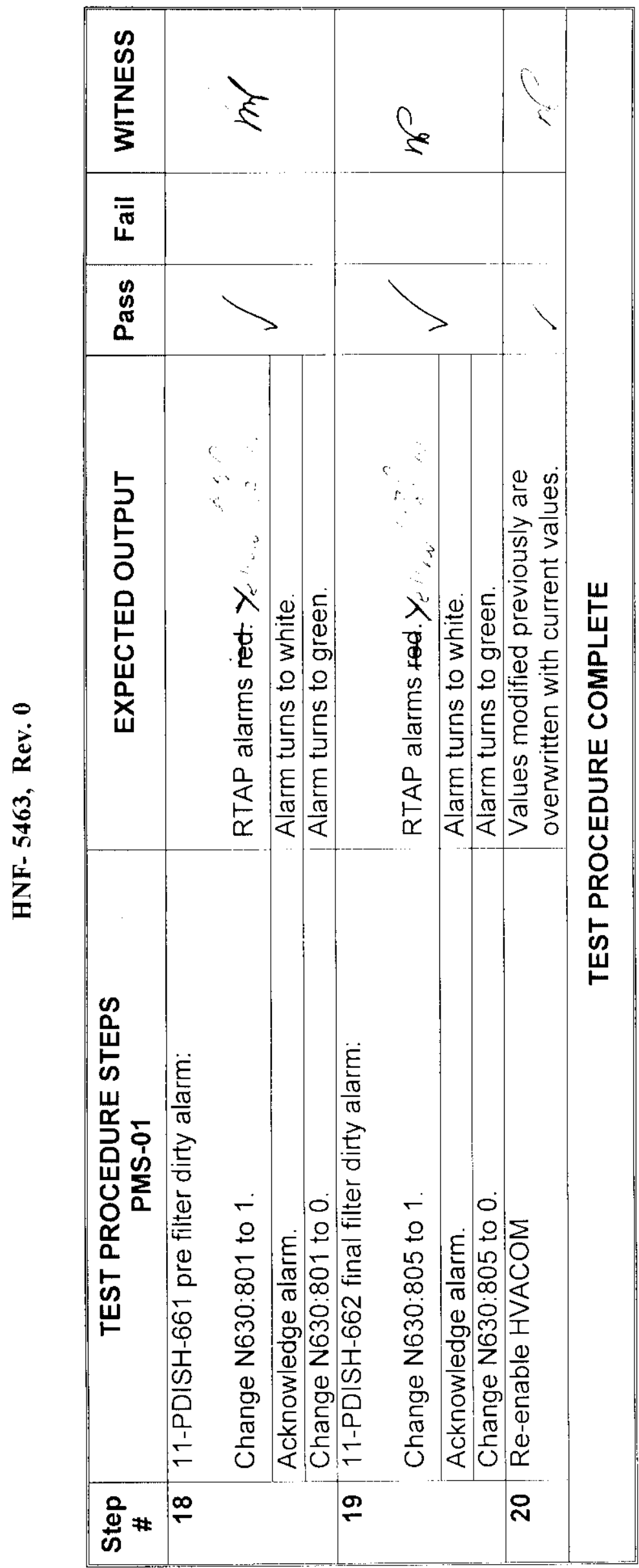

$\stackrel{\sigma}{\sigma}$ 


$$
\text { PMs-ol Step \#6 fige lof } 3
$$

pmstest\% cat backup_test_initial

Script started on Mon Nov 22 09:55:09 1999

pmstest\% s

SQL*Plus: Release 8.0.5.0.0 - Production on Mon Nov 22 9:55:12 1999

(c) Copyright 1998 oracle corporation. A7l rights reserved.

Connected to:

Oracle8 Enterprise Edition Release 8.0.5.0.0 - Production

with the Partitioning and objects options

$\mathrm{PL} / \mathrm{SQL}$ Release 8.0.5.0.0 - Production

SQL> select count (*) from testwaste;

COUNT $(\stackrel{*}{*})$

502

SQL> select count $(*)$ from testconloc;

COUNT (*)

1120

SQL> exit

Disconnected from Oracle8 Enterprise Edition Release 8.0.5.0.0 - Production with the Partitioning and objects options

$\mathrm{PL} / \mathrm{SQL}$ Re?ease 8.0.5.0.0 - Production

pmstest\%

HNF- 5463, Rev. 0, Page 20 


$$
\text { Pms-0) step \#6 puge } 20 \text { F }
$$

pmstest\% cat backup_test_during

Script started on Mon Nov 22 11:08:37 1999

pmstest\% s

SQL*Plus: Release 8.0.5.0.0 - Production on Mon Nov 22 11:8:41 1999

(c) Copyright 1998 oracle Corporation. A71 rights reserved.

Connected to:

Oracle8 Enterprise Edition Release 8.0.5.0.0 - Production

with the Partitioning and objects options

$\mathrm{PL} / \mathrm{SQL}$ Release 8.0.5.0.0 - Production

SQL $>$ select * count $(*)$ from testwaste;

select count $(*)$ from testwaste

ERROR at line 1:

ORA-01115: IO error reading block from file 5 (block \# 96)

ORA-01110: data file 5: '/oral/pmstest/users. 1'

ORA-27041: unable to open file

HP-UX Error: 2: No such file or directory

Additional information: 3

SQL> select count (*) from testcon 7 oc;

select count (*) from testconloc

ERROR at line 1:

ORA-01115: IO error reading block from file 5 (block \# 196)

ORA-01110: data file 5: '/ora1/pmstest/users. 1'

ORA-27041: unable to open file

HP-UX Error: 2: No such file or directory

Additional information: 3

SQL> exit

Disconnected from Oracle8 Enterprise Edition Release 8.0.5.0.0 - Production with the Partitioning and objects options

$\mathrm{PL} / \mathrm{SQL}$ Release 8.0.5.0.0 - Production

pmstest\%

script done on Mon Nov 22 11:09:13 1999

HNF- 5463, Rev. 0,

Page 21 


$$
\text { phos-ol steg } \# \text { b page } 3 \text { of } 3
$$

pmstest\% cat backup_test_final

Script started on Mon Nov 22 11:15:27 1999

pmstest\% s

SQL*Plus: Release 8.0.5.0.0 - Production on Mon Nov 22 11:15:29 1999

(c) Copyright 1998 oracle Corporation. A7l rights reserved.

Connected to:

Oracle8 Enterprise Edition Release 8.0.5.0.0 - Production

with the Partitioning and objects options

$\mathrm{PL} / \mathrm{SQL}$ Release 8.0.5.0.0 - Production

SQL> select count (*) from testwaste;

$\operatorname{COUNT}(*)$

502

SQL> select count (*) from testconloc;

COUNT $(*)$

1120

SQL> exit

Disconnected from oracle8 Enterprise Edition Release 8.0.5.0.0 - Production with the Partitioning and objects options

$\mathrm{PL} / \mathrm{SQL}$ Release 8.0.5.0.0 - Production

pmstest\%

HNF- 5463, Rev. 0, Page 22 


\section{DISTRIBUTION SHEET}

To

Distribution

Project Title $/$ Work Order

WRAP PLANT MANAGEMENT SYSTEM TEST REPORT HNF-5463, Rev. 0

Name

JE Geary

JK Kersten

MP Lane

ME Palmer

CE Taylor

WR Thackaberry

JR Weidert

DOE/RL Reading Room

Engineering Files
From

WRAP Engineering

\begin{tabular}{l} 
MSIN \\
\hline T 4-51 \\
\hline T 4-52 \\
\hline T 4-52 \\
\hline T 4-52 \\
\hline T 4-52 \\
\hline T 4-52 \\
\hline T 4-52 \\
\hline H2 -53 \\
\hline B $1-07$ \\
\hline
\end{tabular}

\begin{tabular}{|l|l} 
Page $1 \quad$ of 1 \\
\hline Date $11 / 23 / 99$ \\
\hline
\end{tabular}

EDT No. 623543

ECN No. N/A

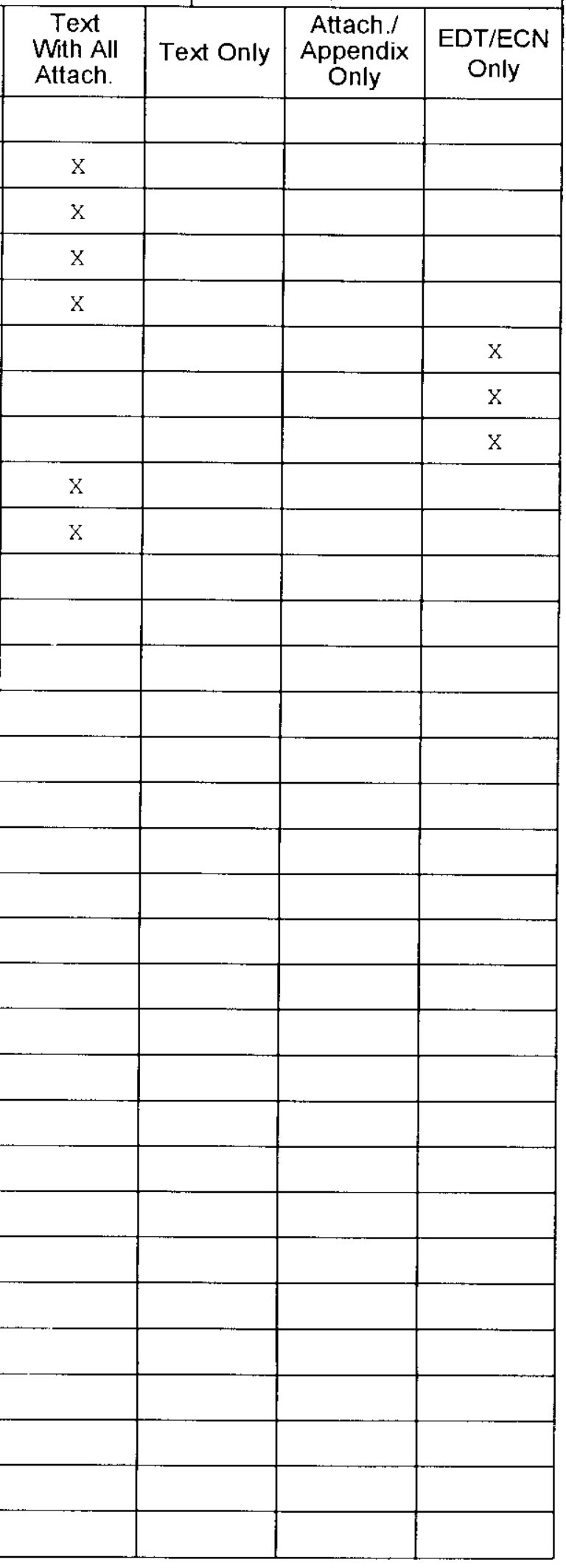

